\title{
Beam dynamics of the racetrack-shape fixed field induction accelerator
}

\author{
Taufik, ${ }^{1,2,3}$ Toshikazu Adachi, ${ }^{2}$ Masayoshi Wake, ${ }^{2}$ and Ken Takayama ${ }^{2}$ \\ ${ }^{1}$ The Graduate University of Advanced Studies (SOKENDAI), Hayama, Kanagawa 240-0193, Japan \\ ${ }^{2}$ High Energy Accelerator Research Organization (KEK), Tsukuba, Ibaraki 305-0801, Japan \\ ${ }^{3}$ National Nuclear Energy Agency of Indonesia (BATAN), Yogyakarta 55281, Indonesia
}

(Received 17 October 2018; published 25 April 2019)

\begin{abstract}
Beam dynamics of the racetrack-shape fixed field induction accelerator for giant cluster ions were established. Linear optics theory and Runge-Kutta particle tracking approaches were developed, and the consistency between two approaches was confirmed. Use of gradient field bending magnets with a reverse field strip and ramping quadrupole doublets were crucial to achieve the orbit stability. A physically limited size of the huge bending magnet in the longitudinal direction generated the inherent closed-orbit distortion that substantially affected the orbit stability. In addition, the inherent field component $B_{s}$ along the orbit coordinate " $s$ " induced horizontal-vertical (H-V) couplings. A possible correction method of this closedorbit distortion was proposed. A net effect of the $\mathrm{H}-\mathrm{V}$ coupling was evaluated. Longitudinal motion with a time-varying transition energy, which is one of characteristic features in the racetrack-shape fixed field induction accelerator, was studied with the help of computer simulations.
\end{abstract}

DOI: 10.1103/PhysRevAccelBeams.22.044001

\section{INTRODUCTION}

The localized energy deposit in a material from an energetic projectile cluster ion is predicted to be substantial. Nonlinear effects during energy deposition, called cluster effects, have been reported [1]. The locally deposited energy density is huge. Such an externally deposited high density of energy has not ever been realized. The instantaneous energy deposition and rapid cooling in the target material associated with the impact of a giant cluster ion bunch is expected to induce new material phases that have never been observed under natural conditions on Earth. A research forum pursuing these new material phases and their applications has recently been founded in Japan [2]. An international collaboration to develop a high-chargestate giant cluster ion source was initiated in Orsay, France this winter [3].

Experimental studies using low-energy cluster ions started at the Institute of Nuclear Physics of Orsay [4], where $30 \mathrm{MeV} \mathrm{C}_{60}$ cluster ions are obtained by the electrostatic tandem accelerator, and various important features, such as the cluster effect, have been revealed [5,6]. The Takasaki Ion Accelerators for Advanced Radiation Application electrostatic accelerator facility, Takasaki, Japan, subsequently demonstrated the

Published by the American Physical Society under the terms of the Creative Commons Attribution 4.0 International license. Further distribution of this work must maintain attribution to the author(s) and the published article's title, journal citation, and DOI. acceleration of various cluster and molecular ions (B, C, $\mathrm{O}, \mathrm{Al}, \mathrm{Si}, \mathrm{Cu}, \mathrm{Au}, \mathrm{LiF}$, and $\mathrm{AlO}$ ) using a $3 \mathrm{MV}$ tandem accelerator [7]. However, there is a technical limitation in realizing high-energy giant cluster ions by electrostatic acceleration due to high-voltage breakdown. To obtain giant cluster ions with higher energies, circular acceleration is necessary. The rf acceleration based on an $\mathrm{rf}$ system consisting of an rf cavity and rf source, such as a tetrode, is not suitable because of the bandwidth limitation of the $\mathrm{rf}$ devices. The revolution frequency of giant cluster ions, which have large mass-to-charge ratios $(A / Q>720)$, is too small (order of kilohertz) at injection and increases dynamically with acceleration. This dynamic change in the revolution frequency can be followed by induction acceleration. After a slow-cycling induction synchrotron was demonstrated using the KEK proton synchrotron in 2007 [8], the fast-cycling induction synchrotron showed the acceleration from $50 \mathrm{kHz}$ to $1 \mathrm{MHz}$ in 2013 [9]. Based on these results, a racetrack-shape fixed field induction accelerator (RAFFIA) was proposed in 2015 as a unique solution to achieve repeated acceleration of giant cluster ions [10]. The most important beam guiding device is the four $90^{\circ}$ bending magnets with a gradient field and reverse field strip at the front.

Although an overview of RAFFIA has been published [10], its details have not been described. The orbit stability of RAFFIA is a most important issue from the beam dynamics point of view. The RAFFIA resembles an electron microtron with $90^{\circ}$ bending magnets, where two straight sections are occupied by the quadrupole magnets for focusing and a high-gradient microwave acceleration 
tube that looks like a linac. The total revolution number of a beam bunch in an electron microtron is limited because of high-gradient acceleration, that is, 20-40 turns. In contrast, a giant cluster ion bunch performs a circulation of more than 1000 turns because of the relatively small acceleration voltage of $10-20 \mathrm{kV}$. This number of turns may be similar to that in a cyclotron rather than a synchrotron. However, the aspect that most distinguishes the RAFFIA from a cyclotron or synchrotron is that the circumference of the RAFFIA gradually changes with acceleration. It is noted that the change in the orbit circumference is not so large as that in a cyclotron, and essential features of motion there may be rather similar to that in a synchrotron.

Important concepts in the synchrotron orbit theory, such as lattice functions or betatron tune, are discussed in Ref. [10], assuming that the change in circumference is moderate and the linearized fields around the time-varying ideal orbit determine the essential features of the particle motion around the ideal orbit. These assumptions suggest a pseudolinear orbit theory for the RAFFIA. The change in circumference with acceleration is caused by the varying orbit in the bending magnet region. This also demands a dynamic change in the $k$ value of the focusing magnets. The effects of the fringing fields of the bending magnet are nonnegligible because of the fixed fields. The ideal orbit cannot be discussed without considering these effects; thus, the discussion in Ref. [10] is too simplified, because the effects of the fringing fields on the ideal orbit or reference orbit were ignored.

In order to establish the linear beam dynamics theory for the RAFFIA, the original particle tracking code, where the 3D magnetic field profile in the fixed field bending magnet is implemented, has been developed. Many particle tracking simulation codes for the fixed field accelerator such as a cyclotron, microtron, and fixed field alternating gradient are known. Unfortunately, there is no code to universally correspond to all fixed field accelerators, because their magnetic field configuration strongly depends on an individual type. In this sense, the present code is specific for the RAFFIA.

In the linear theory, a bending field of a magnet with infinite length is assumed (ideal bending field). The reference orbit of each turn is obtained using the simulation results. Next, the bending fields are linearized around this reference orbit, resulting in the local field gradient. Using the local gradient along the orbit axis, $s$, on the reference orbit, the linear transfer matrix for a desired segment is derived. Once the linear matrix for the entire region of the circumference is established, the linear stability condition tells us the optimized $k$ values of the set of focusing quadrupole magnets placed on two straight sections. Thus, the crucial beam dynamics information, such as the lattice function, momentum dispersion function, betatron tunes, and momentum compaction factor, is evaluated. All the terms change with acceleration.
The utilization of opposite magnetic fields generated in the gradient field and reverse field strip of the bending magnet causes the particle orbit of the RAFFIA pass through a nonuniform magnetic field. This nonuniform magnetic field contains a field component $B_{s}$ in the $s$ direction which generates a horizontal-vertical $(\mathrm{H}-\mathrm{V})$ coupling. This kind of $\mathrm{H}-\mathrm{V}$ coupling does not appear in the uniform field bending magnet in a synchrotron. Therefore, the effect of the inherent $\mathrm{H}-\mathrm{V}$ coupling on the particle motion must be evaluated.

There is another important source perturbing on the beam orbit that is never seen in a synchrotron. This originates from the finite physical length along the longitudinal direction of the realistic bending magnet, where the field profile is nonuniform in the longitudinal direction; the profile is symmetrical around the magnet center in the longitudinal direction but necessarily falls down toward both edges. The reference orbit gradually shifts in the longitudinal direction. Thus, the deviation from the uniform ideal magnetic flux density becomes a source of the timevarying closed-orbit distortion (COD). This feature is intrinsic in the RAFFIA, because the nonuniformity of the magnetic field profile is, in principle, unavoidable, even with shimming.

Other important issues with the RAFFIA are acceleration and confinement in the longitudinal phase space of a giant cluster ion bunch. The acceleration itself is relatively easy, because there is no magnetic-field ramping, and we assume a constant acceleration voltage per turn. However, the barrier trapping must be simulated considering the change in the momentum compaction factor with acceleration. To understand the particle behavior in the longitudinal direction, the macroparticle simulation code has also been developed. The loss mechanism in the longitudinal phase space is discussed. Finally, remaining beam dynamics related to the space-charge effect issues are addressed.

\section{OUTLINE OF THE INDUCTION MICROTRON}

The RAFFIA for $\mathrm{C}_{60}(A=720, Z=10)$ cluster ions is used as an example [11], where the injection energy and extraction energy are 10 and $144 \mathrm{MeV}$, respectively, and the circumference changes from 49.1 to $54.3 \mathrm{~m}$. An induction acceleration voltage of $10 \mathrm{kV}$ per turn requires a total turn number of 1339. For its reality [12], the size of the RAFFIA has been chosen so as to fit the existing space for the KEK digital accelerator.

The RAFFIA lattice consists of four $90^{\circ}$ bending magnets $\left(\mathrm{BM}_{i}, i=1-4\right)$ and four doublet $\mathrm{Q}$ magnets $\left(\mathrm{QF}_{i} / \mathrm{QD}_{i}\right.$, $i=1-4)$ occupying the upper and lower straight section (Fig. 1). The injection system and induction acceleration system are also placed on the lower straight section. The giant cluster ion beam delivered from the ion source embedded in the high-voltage platform is accelerated using the $1 \mathrm{MV}$ induction linac and injected into the RAFFIA ring. The injection or exit angle from the bending magnet is 


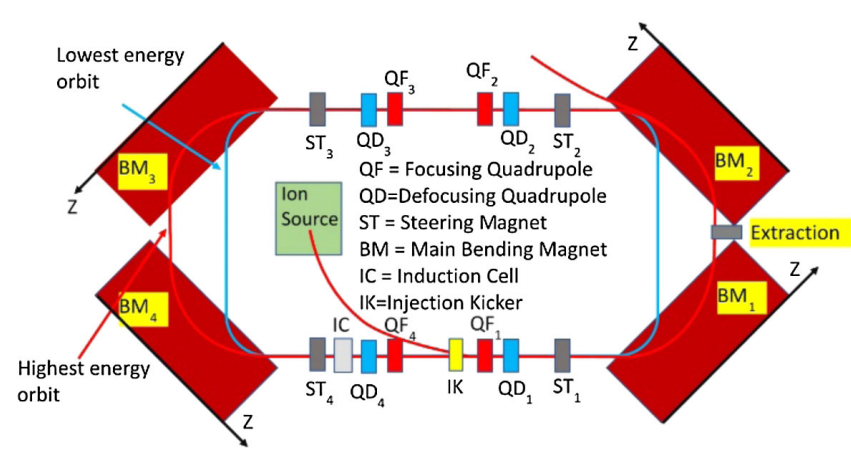

FIG. 1. Schematic view of the RAFFIA.

$45^{\circ}$. The orbit in the straight sections does not depend on the particle energy. Steering magnets must be installed on the straight sections, as described below. In addition, a kicker system for extraction is placed between bending magnets $\mathrm{BM}_{1}$ and $\mathrm{BM}_{2}$. It has been confirmed that the inward kick by the kicker can give a suitable extraction orbit from the RAFFIA ring at the downstream edge of $\mathrm{BM}_{2}$, although its details are not discussed in this paper.

\section{A. Beam guiding (bending magnet)}

The bending magnet for the RAFFIA performs $90^{\circ}$ bending and focusing in the vertical direction. The injection or extraction (edge) angle of $45^{\circ}$ induces extremely large edge defocusing in the vertical direction, which substantially affects the beam motion, as discussed in Ref. [10]. The reverse field strip at the front edge and field gradient on the main pole are essential for mitigating defocusing in the vertical direction and achieving orbit stability [10]. The characteristics of focusing and defocusing in the vertical direction through the bending magnet region are shown in Fig. 2.

The bending magnet is designed by using ANSYS 3D®. $B_{y}, \frac{d B_{y}}{d X}$, and $\frac{d B_{y}}{d Z}$ data on the median plane of the magnet gap are imported from the ANSYS 3D calculation result in a mesh size of $2 \mathrm{~cm}$ in the $X-Z$ plane. The designed bending

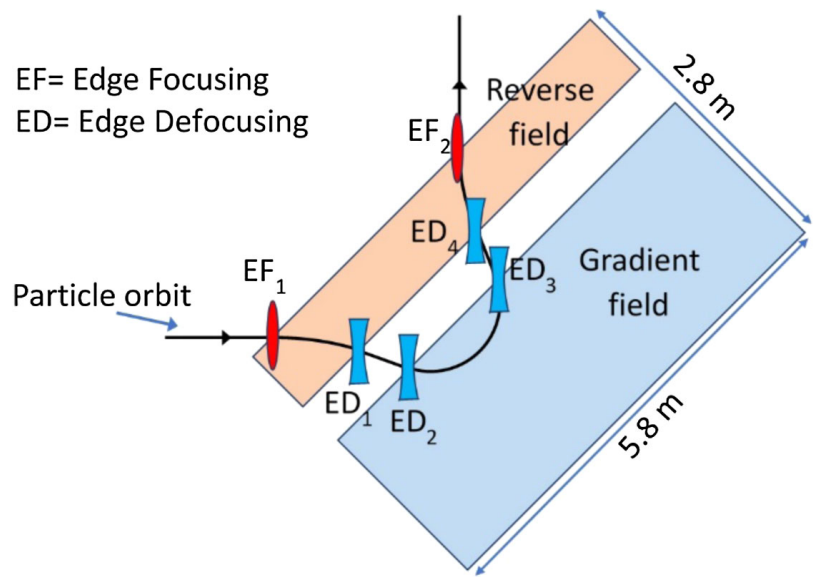

FIG. 2. Vertical edge focusing in the bending magnet.

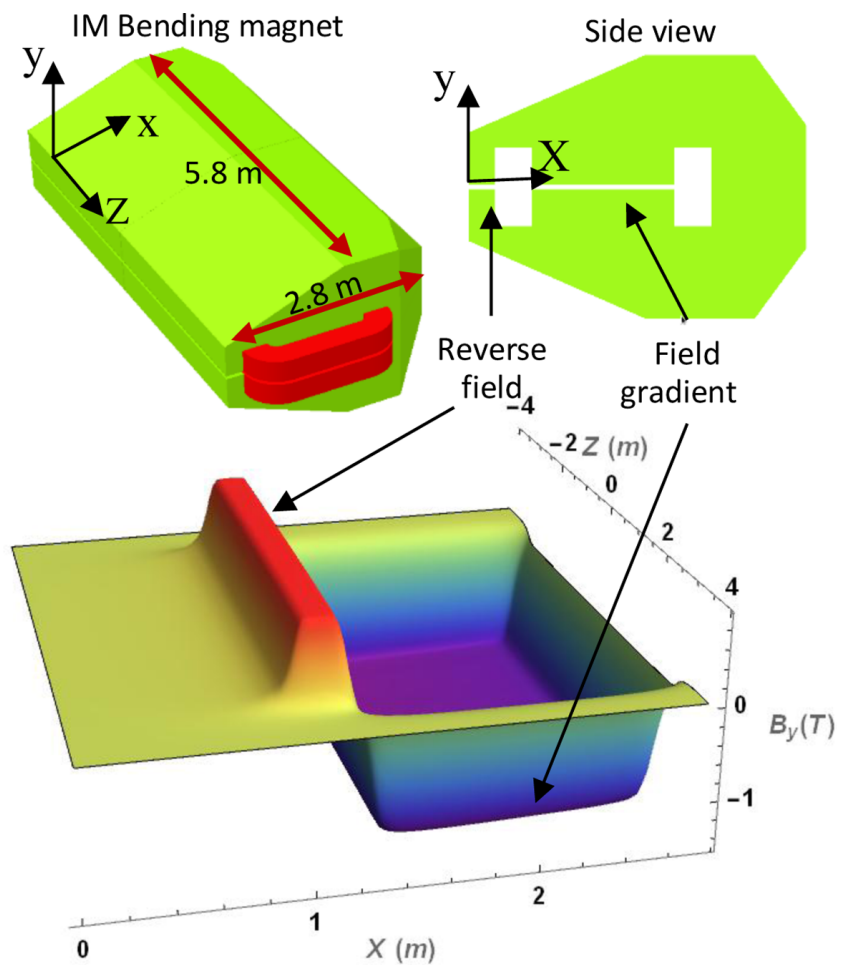

FIG. 3. Bending magnet and its bending field component $B_{y}$ distribution on the median plane.

magnet and magnetic field distribution on the median plane are shown in Fig. 3. The main bending field and reverse field are excited with the expected flux densities in the different gaps by a single pair of excitation coils. A fraction of the magnetic flux in the main gap returns to the core front rather than to the return yoke due to the difference in magnetic resistance through two magnetic flux loops. Recently, this idea has been experimentally confirmed using a prototype bending magnet [13]. The magnet field calculations indicate a peak flux density of $0.98 \mathrm{~T}$ in the front gap and $-1.5 \mathrm{~T}$ in the main bending region. The magnet is $5.8 \mathrm{~m}$ long and $2.8 \mathrm{~m}$ wide so as to cover the particle orbit up to the extraction energy.

\section{B. Acceleration (induction device)}

The RAFFIA will use induction acceleration cells similar to those of the KEK digital accelerator, as described in Ref. [9]. Three cells will be used for acceleration, and one cell will be used for barrier trapping. Individual cells will be energized by a newly developed switching power supply, which uses four $3.3 \mathrm{kV} \mathrm{SiC-MOSFET} \mathrm{switching} \mathrm{elements}$ in the full-bridge circuit (Fig. 4).

The induction cells generate an acceleration voltage $\left(V_{a c}\right)$ and barrier voltage $\left(V_{b b}\right)$ for each acceleration cycle (Fig. 5). For confinement, two $V_{b b}$ pulses of pulse length $\phi_{\text {pulse }}$ are generated with a phase duration $(\Delta \phi)$ between the negative pulse and positive pulse. The beam bunch is accelerated only in the beam confinement region. The length of a beam bunch is varied by controlling the trigger 


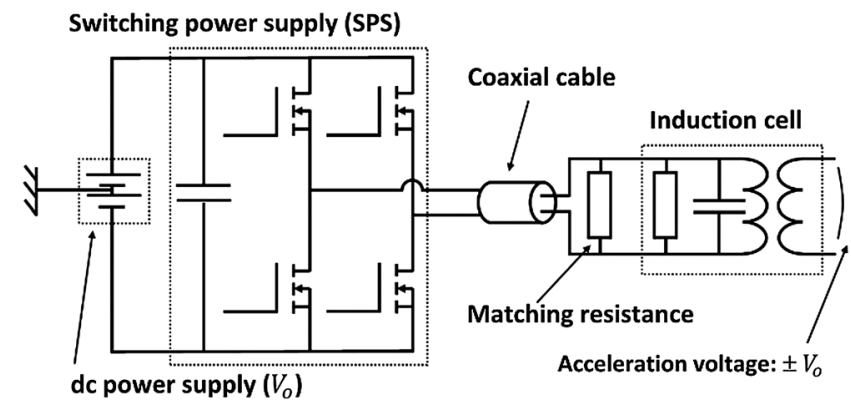

FIG. 4. Induction cell driver circuit.

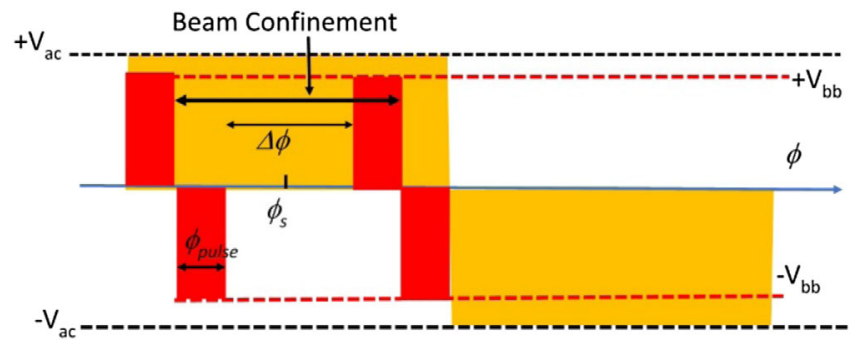

FIG. 5. Induction acceleration voltages.

signal of the barrier voltage pulses. This method should be effective for extracting the bunch with a desired length by the kicker system at the end of acceleration, as repeatedly emphasized in references related to induction acceleration.

\section{TRANSVERSE MOTION}

The transverse motion of the particles around the reference orbit, known as betatron motion, is defined by Hill's equation

$$
Y^{\prime \prime}+K(s) Y=0,
$$

where $Y$ is the transverse coordinate, $s$ is the longitudinal coordinate, and $K(s)$ is the restoring force coefficient. Hill's equation can be solved by a linear optics approach using a transfer matrix. In the transfer matrix form, the transverse motion calculation is defined by

$$
\bar{Y}_{s_{1}}=M_{s_{0} \rightarrow s_{1}} \cdot \bar{Y}_{s_{0}},
$$

where $\bar{Y}$ is the vector for the transverse position in phase space and $M$ is a $2 \times 2$ transfer matrix. For the orbit stability of the circular accelerator, a one-turn transfer matrix $\left(M_{T}\right)$, which is obtained by multiplying the sequence of $M$, must satisfy the stable condition where

$$
\left|\frac{\operatorname{Trace}\left(M_{T}\right)}{2}\right| \leq 1
$$

A stable orbit indicates that the particle motion in one turn is always confined around a closed orbit.
The transfer matrix represents the optical properties of the devices around the reference orbit. Therefore, the reference orbits for the stable motion must be known to determine the transfer matrix. As the particle orbits in the RAFFIA gradually change with acceleration, the closed orbit must be found for every turn. A particle tracking code is developed to find the closed orbit.

\section{A. Runge-Kutta particle tracking}

In a global coordinate system, the particle motion under the influence of an electromagnetic field is defined by the following Lorentz equation:

$$
\begin{aligned}
\boldsymbol{F}_{L} & =\frac{d \boldsymbol{p}}{d t}=m \gamma \frac{d^{2} \boldsymbol{r}}{d t^{2}}=q\left(\boldsymbol{E}(\boldsymbol{r})+\frac{\boldsymbol{p} \times \boldsymbol{B}(\boldsymbol{r})}{m \gamma}\right), \\
\frac{d \boldsymbol{r}}{d t} & =\frac{\boldsymbol{p}}{m \gamma},
\end{aligned}
$$

where $\boldsymbol{F}_{L}$ is the Lorentz force, $\boldsymbol{p}$ is the momentum, $t$ is the time, $m$ is the particle mass, $\gamma$ is the Lorentz factor, $\boldsymbol{r}$ is the position vector, $q$ is the particle charge, $\boldsymbol{E}$ is the electric field, and $\boldsymbol{B}$ is the magnetic field. The Lorentz equation is a second-order ordinary differential equation, which is difficult to solve analytically in three dimensions. Therefore, the Lorentz equation is solved numerically with a sixthorder Runge-Kutta method. In this sixth-order Runge-Kutta method, seven evaluation stages are used at each step with Runge-Kutta coefficients written in the following Butcher table [14]:

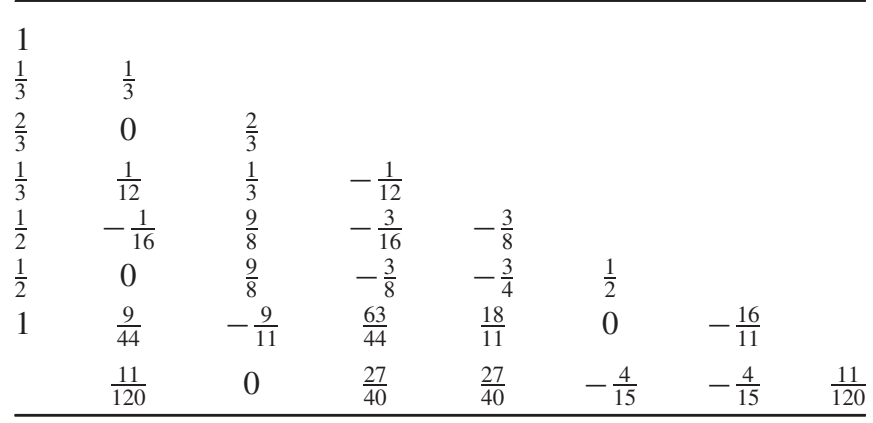

The sixth-order Runge-Kutta solution for Eq. (4) is shown in the Appendix.

The flowchart of the particle tracking code is shown in Fig. 6. The Runge-Kutta method is used only in the bending magnets and quadrupole magnets section, whereas a kick approximation is used in the steering magnet and acceleration section. The magnetic field data in the quadrupole are assumed an ideal field which is determined by its field gradient. Meanwhile, the magnetic fields in the bending magnet are obtained from the expansion of a discrete magnetic field data on the median plane. The expansion of the magnetic fields beyond the grid points on the median plane is obtained in a way of linear interpolation between adjacent points. $B_{y}$ in the upper and lower areas of the median plane is assumed to be equal to $B_{y}$ on the 


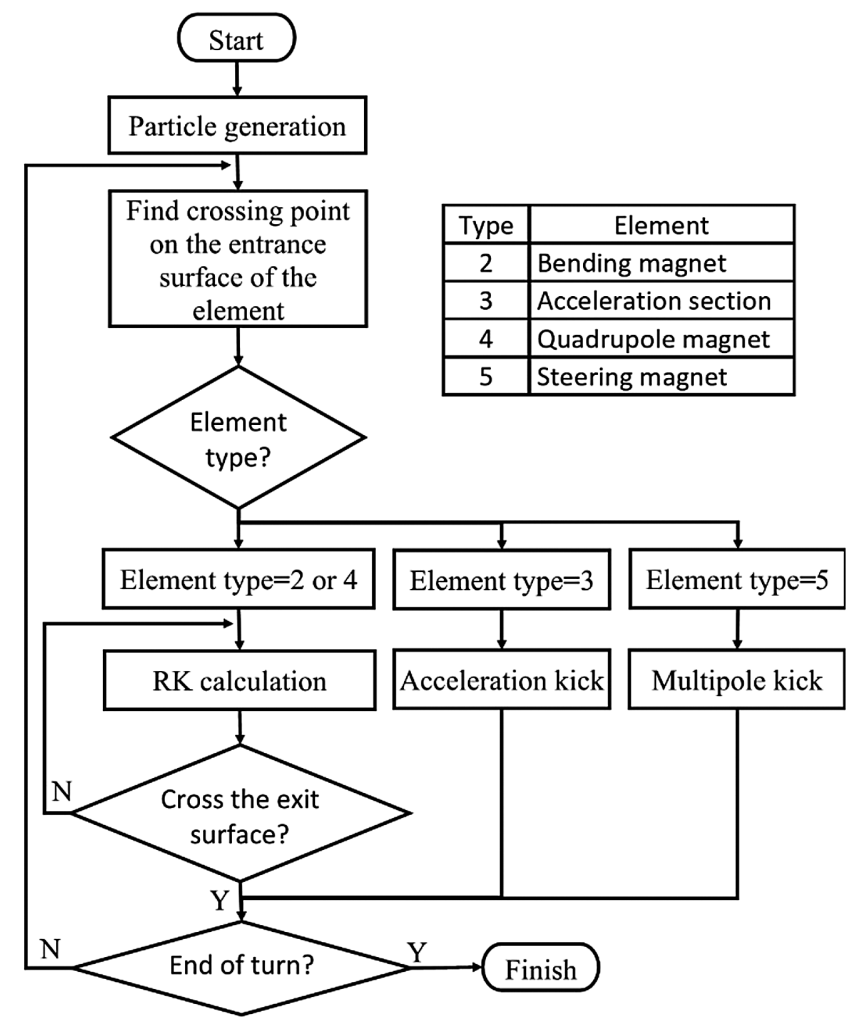

FIG. 6. Flowchart of the particle tracking code.

median plane. Meanwhile, $B_{X}$ and $B_{Z}$ in the upper and lower areas of the median plane are expressed in the forms expanded to the first order of $y$. The reference orbit is calculated, assuming that the bending magnet fields are uniform along the $Z$ direction and the field itself at the center of magnet (origin of the $Z$ direction) obtained from the ANSYs 3D calculation. The field is called an ideal field in the paper. The initial position of the particle is defined at the injection kicker section.

Typical tracking results in the global coordinate system are shown in Fig. 7. One-turn transfer matrices can be defined every turn by running $N$-particle tracking. By generating $N$ particles in the phase space, the elements of the transfer matrix are given in terms of the least squares fit by

$$
\begin{aligned}
\left(\begin{array}{c}
Y_{k} \\
Y_{k}^{\prime}
\end{array}\right)_{n+1}=M_{T}\left(\begin{array}{l}
Y_{k} \\
Y_{k}^{\prime}
\end{array}\right)_{n}=\left(\begin{array}{ll}
m_{11} & m_{12} \\
m_{21} & m_{22}
\end{array}\right)\left(\begin{array}{c}
Y_{k} \\
Y_{k}^{\prime}
\end{array}\right)_{n}, \\
\chi^{2}=\sum_{k=1}^{N}\left\{\left(Y_{k}^{(n+1)}-m_{11} Y_{k}^{(n)}-m_{12} Y_{k}^{\prime(n)}\right)^{2}\right. \\
\left.+\left(Y_{k}^{(n+1)}-m_{21} Y_{k}^{(n)}-m_{22} Y_{k}^{\prime(n)}\right)^{2}\right\}=\min ,
\end{aligned}
$$

where $k$ is the particle number and $n$ is the turn number.

A stable orbit is obtained if the transfer matrix satisfies Eq. (3). The quadrupole doublets are the only parameter

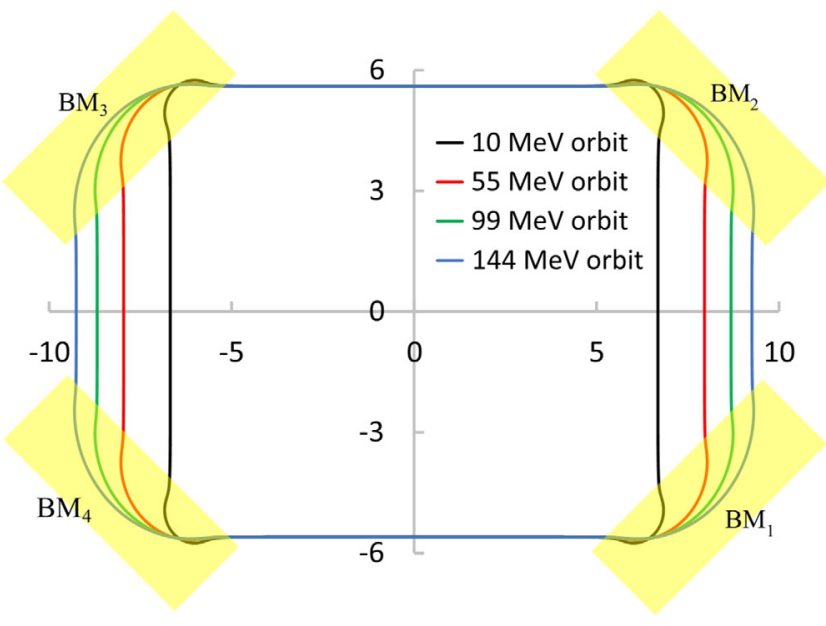

FIG. 7. Typical tracking result.

that can be adjusted to obtain stable closed orbits. Figure 8 shows the field gradient of the optimized quadrupole doublet. This ramping pattern should be performed in $17.66 \mathrm{~ms}$. The tune diagram shown in Fig. 9 does not pass the integer resonances.

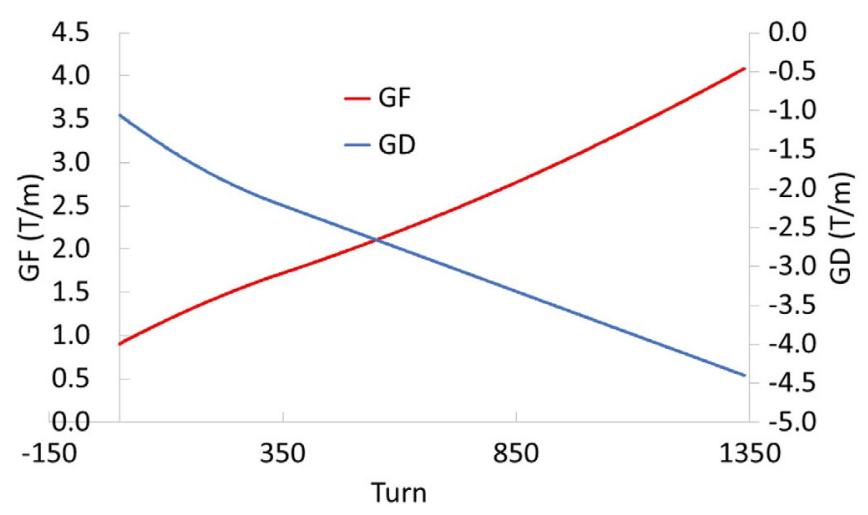

FIG. 8. Field gradient of the optimized quadrupole doublets.

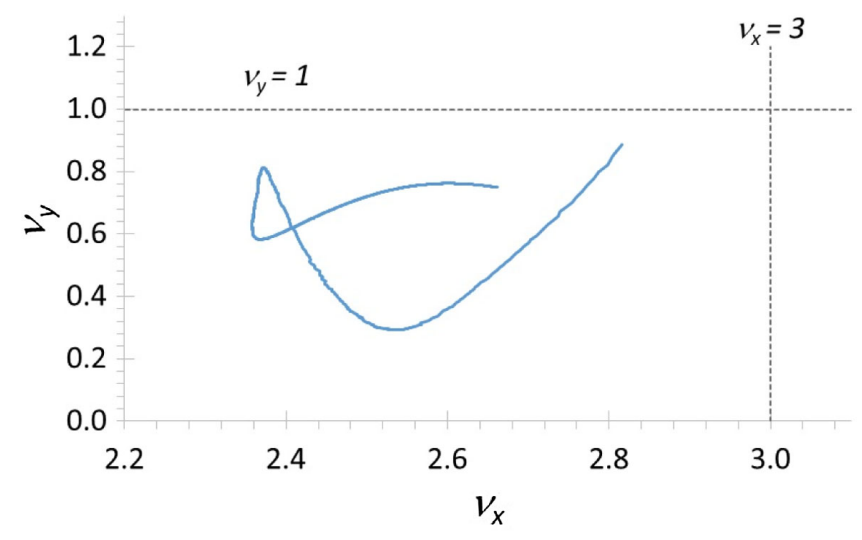

FIG. 9. Tune diagram of the RAFFIA. 


\section{B. Linear optics particle tracking}

The linear optics approach uses a closed orbit obtained by particle tracking as a reference orbit. The data for the transfer matrix calculation along the closed orbit, such as magnetic rigidity and $\frac{d B_{y}}{d x}$, are collected at each step size. From these data, the one-turn restoring force coefficient $K(s)$ can be calculated. Figure 10 shows $K(s)$ in the half lattice at the injection energy.

Ampere's law suggests the magnetic field component in the longitudinal direction, $B_{s}$, from the no-zero gradient the transverse magnetic field components, $\partial H_{y} / \partial X, \partial H_{x} / \partial Y$, yielding the coupling among the transverse motion. The coupling effects are described in the linear perturbed betatron equations

$$
\begin{aligned}
x^{\prime \prime}+\left(\frac{1}{\rho^{2}}-K_{y}(s)\right) x & =-\frac{B_{s}}{(B \rho)} y^{\prime}, \\
y^{\prime \prime}+K_{y}(s) x & =\frac{B_{s}}{(B \rho)} x^{\prime},
\end{aligned}
$$

where $\rho$ is the bending radius, $K_{y}(s)$ is the restoring coefficient in the vertical direction, and $(B \rho)$ is the magnet rigidity. Here, $\frac{1}{\rho^{2}}-K_{y}(s)=K_{x}(s)$ is the restoring coefficient in the horizontal direction. The solution of the perturbed Hill's equation can be approximated by

$$
\left(\begin{array}{c}
x \\
x^{\prime} \\
y \\
y^{\prime}
\end{array}\right)_{s_{2}}=\left(\begin{array}{cccc} 
& M_{x} & 0 & 0 \\
0 & 0 & & -\frac{1}{B \rho} B_{s}(s) \Delta s \\
0 & \frac{1}{B \rho} B_{s}(s) \Delta s & M_{y}
\end{array}\right)\left(\begin{array}{c}
x \\
x^{\prime} \\
y \\
y^{\prime}
\end{array}\right)_{s_{1}},
$$

where $M_{x}$ and $M_{y}$ are $2 \times 2$ transfer matrices in the horizontal and vertical directions, respectively, and $\Delta s$ is the small step size.

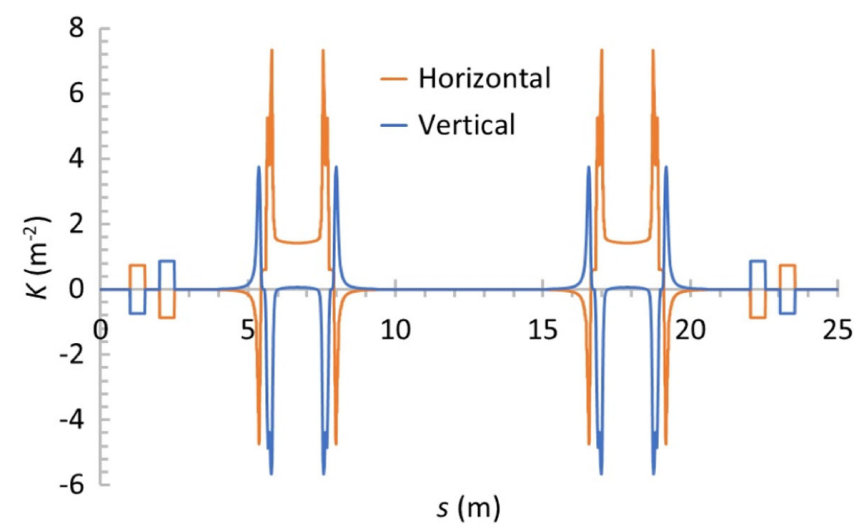

FIG. 10. $K$ value at the injection energy in the half lattice of the RAFFIA.
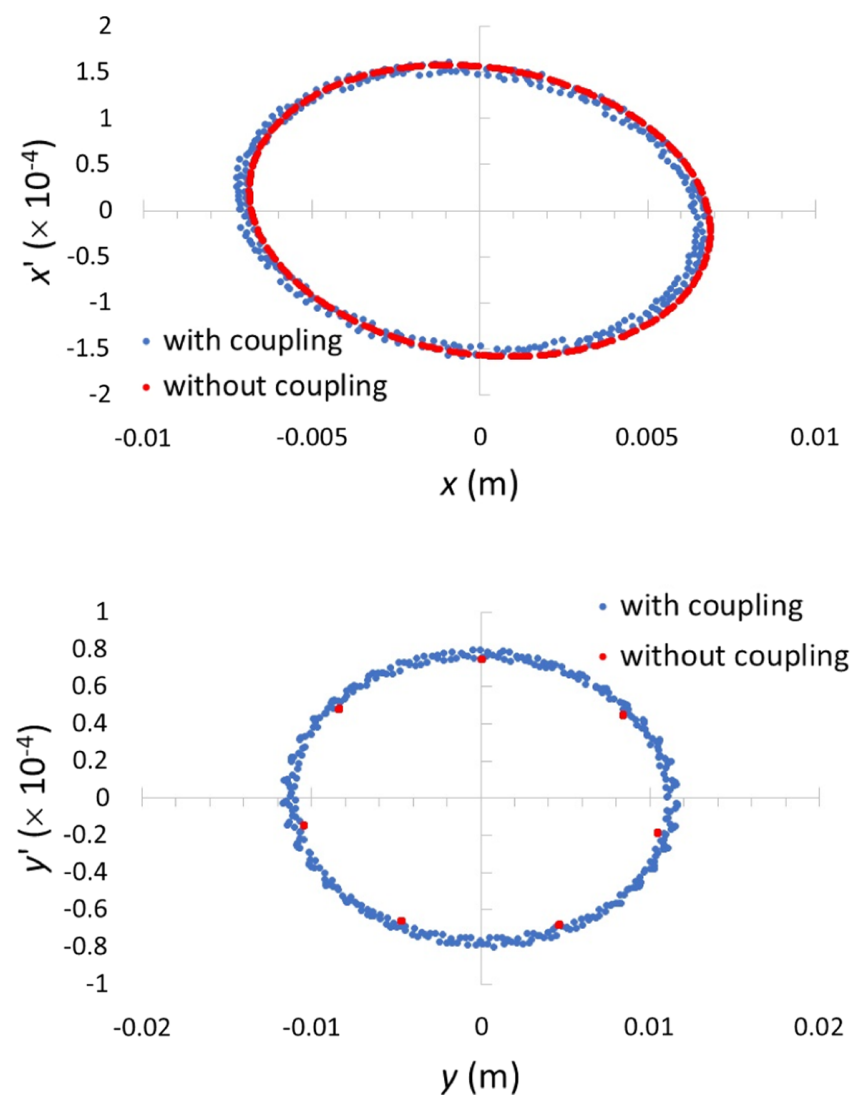

FIG. 11. Poincaré map obtained over 350 turns by using linear optics approach in the horizontal (upper) and vertical (lower) direction.

The effect of the horizontal-vertical $(\mathrm{H}-\mathrm{V})$ coupling in the transverse motion is shown on the Poincaré map over 350 turns. Poincare mapping of the particle motions based on Eq. (7) is shown in Fig. 11. The phase plot of the single particle motion without coupling moves along the ellipse circumference, as expected. Meanwhile, in the case with coupling, the phase plot scatters a bit around the ellipse circumference. In the Runge-Kutta simulation approach, the particle motion calculation automatically includes the $\mathrm{H}-\mathrm{V}$ coupling effect. The comparison of results by both approaches is shown in Fig. 12. Both approaches seem to be similar. The deviation from the noncoupling result is around $2 \mathrm{~mm}$ for the horizontal motion and much smaller for the vertical direction. Anyway, the result indicates that the contribution of $\mathrm{H}-\mathrm{V}$ coupling is relatively small.

\section{Lattice function}

\section{Beta function}

The beam size is important in designing an accelerator, and one of the contributions to the beam size is defined by $(\varepsilon \beta)^{1 / 2}$, where $\varepsilon$ is the emittance and $\beta$ is the beta function. The beta function determines the beam envelope, which describes the outermost betatron motion of the beam. To be derived from Hill's equation, $\beta$ depends on the lattice of the 

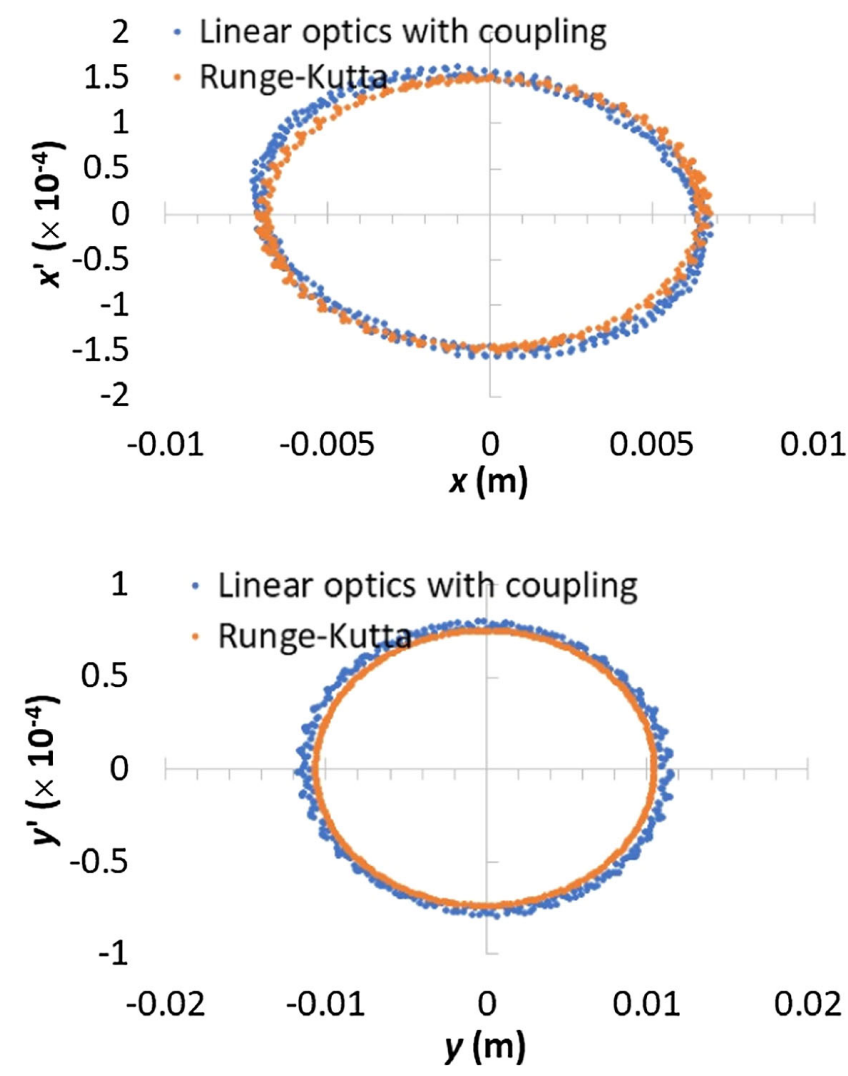

FIG. 12. Poincaré map of a particle motion with H-V coupling in the horizontal (upper) and vertical (lower) direction.

machine. $\beta$ and the other Twiss parameters $(\alpha$ and $\gamma$ ) are calculated from the transfer matrix by

$$
\left(\begin{array}{l}
\beta \\
\alpha \\
\gamma
\end{array}\right)_{s_{2}}=\left(\begin{array}{ccc}
m_{11}^{2} & -2 m_{11} m_{12} & m_{11}^{2} \\
-m_{21} m_{11} & 1+2 m_{12} m_{21} & -m_{12} m_{22} \\
m_{21}^{2} & -2 m_{22} m_{21} & m_{22}^{2}
\end{array}\right)\left(\begin{array}{l}
\beta \\
\alpha \\
\gamma
\end{array}\right)_{s_{1}},
$$

where $m$ is the component of the $2 \times 2$ transfer matrix $M$.

The beta functions of the RAFFIA at the injection and extraction energy are shown in Fig. 13. The vertical beam envelope $\left(\beta_{V}\right)$ is much bigger than that for the horizontal beam envelope $\left(\beta_{H}\right)$. The horizontal and vertical beam envelopes are maximum in the $\mathrm{QF}$ and $\mathrm{QD}$, respectively. At the injection energy, the values of the $\beta_{H}$ maximum, $\beta_{H}$ minimum, $\beta_{V}$ maximum, and $\beta_{V}$ minimum are 47.097, $0.038,327.62$, and $1.550 \mathrm{~m}$, respectively. Meanwhile, at the injection energy, the values of the $\beta_{H}$ maximum, $\beta_{H}$ minimum, $\beta_{V}$ maximum, and $\beta_{V}$ minimum are 15.515, $1.99,305.481$, and $1.752 \mathrm{~m}$, respectively. Figure 13 also shows that the beam envelope at the extraction energy is smaller than the injection energy.
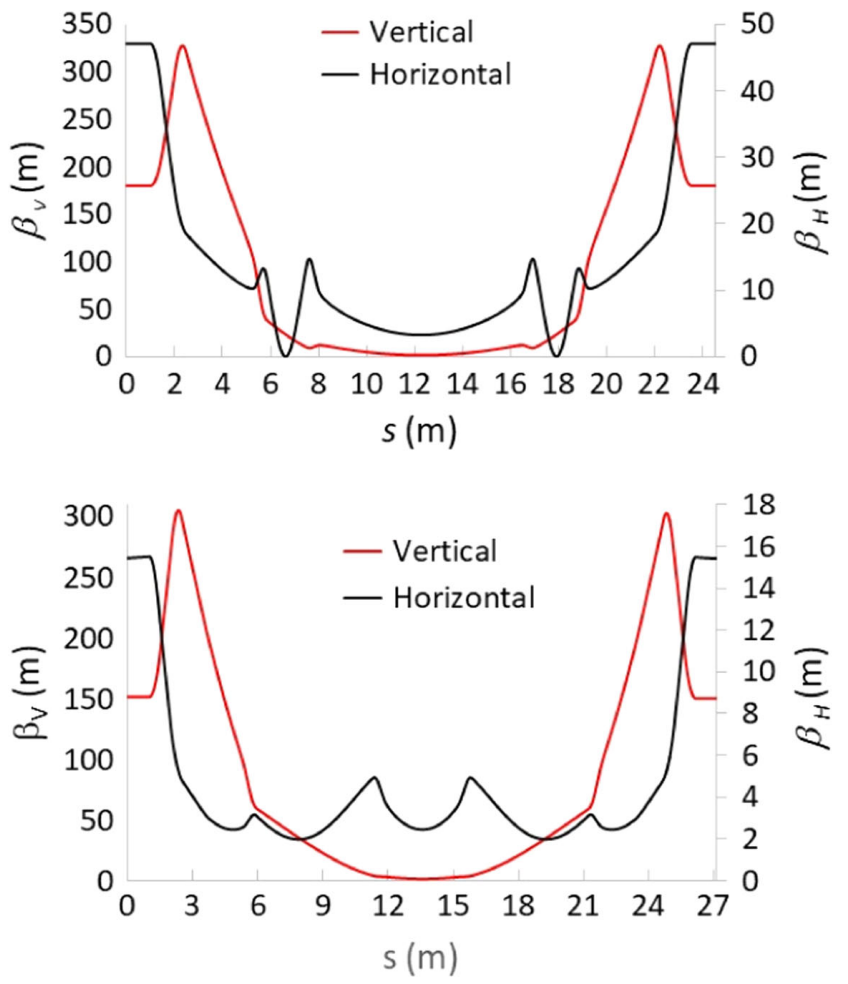

FIG. 13. Beta function in the half lattice of the RAFFIA at the injection (upper) and extraction energy (lower).

\section{Dispersion function}

The particles with $\Delta p / p$ perform their horizontal betatron oscillations around a different orbit from the reference particle. This motion is described by Eq. (1) with additional $(\Delta p / p) / \rho$ in the right-hand side of the equation. With this additional term, Eq. (1) becomes an inhomogeneous differential equation. The general solution of the equation is

$$
x(s)=x_{\beta}(s)+x_{e q}(s),
$$

where $x_{\beta}$ is the homogeneous solution that represents the betatron motion and $x_{e q}$ is the particular solution that represents the displacement of the betatron oscillation center.

The particular solution of the inhomogeneous equation is given by

$$
\begin{aligned}
D(s)= & \frac{\sqrt{\beta(s)}}{2 \sin (\mu / 2)} \int_{s}^{s+C}\left(\frac{1}{\rho\left(s^{\prime}\right)}\right) \sqrt{\beta\left(s^{\prime}\right)} \cos \\
& \times\left[\mu / 2-\left|\Psi(s)-\Psi\left(s^{\prime}\right)\right|\right] d s^{\prime},
\end{aligned}
$$

where dispersion function $D=x_{e q} /(\Delta p / p), \mu$ is the phase advance, and $\Psi$ is the betatron phase. The dispersion function can also be calculated with a $3 \times 3$ transfer matrix by 


$$
\left(\begin{array}{c}
D \\
D^{\prime} \\
1
\end{array}\right)_{s_{2}}=\left(\begin{array}{ccc}
m_{11} & m_{12} & m_{13} \\
m_{21} & m_{22} & m_{23} \\
0 & 0 & 1
\end{array}\right)\left(\begin{array}{c}
D \\
D^{\prime} \\
1
\end{array}\right)_{s_{1}},
$$

where $D^{\prime}$ is the derivative of $D$ with respect to $s$ and $m_{13}$ and $m_{23}$ are matrix components from the particular solution. The dispersion function is calculated by Eq. (11) from the linear optics particle tracking.

In the particle tracking simulation, the dispersion function can be approximated by comparing a reference particle orbit with an off-momentum particle orbit. Both particle orbits are obtained by running the particle tracking code. The distance between the reference orbit and off-momentum orbit is defined as $x_{e q}$. Multiturn data without acceleration are used to correct the small residual oscillation.

The dispersion functions of the RAFFIA at the injection and extraction energy are shown in Fig. 14. The dispersion functions from the linear optics and particle tracking simulations show almost consistent results. The discrepancy may come from the kick approach in the linear optics when calculating the $\mathrm{H}-\mathrm{V}$ coupling, as discussed in the previous section. Figure 14 shows that the dispersion function at the injection energy is much smaller than at the extraction energy. We previously obtained the maximum $\Delta p / p$ of $0.41 \%$ from injecting carbon ions into the fast-cycling induction synchrotron (KEK digital accelerator) [15]. Assuming the maximum
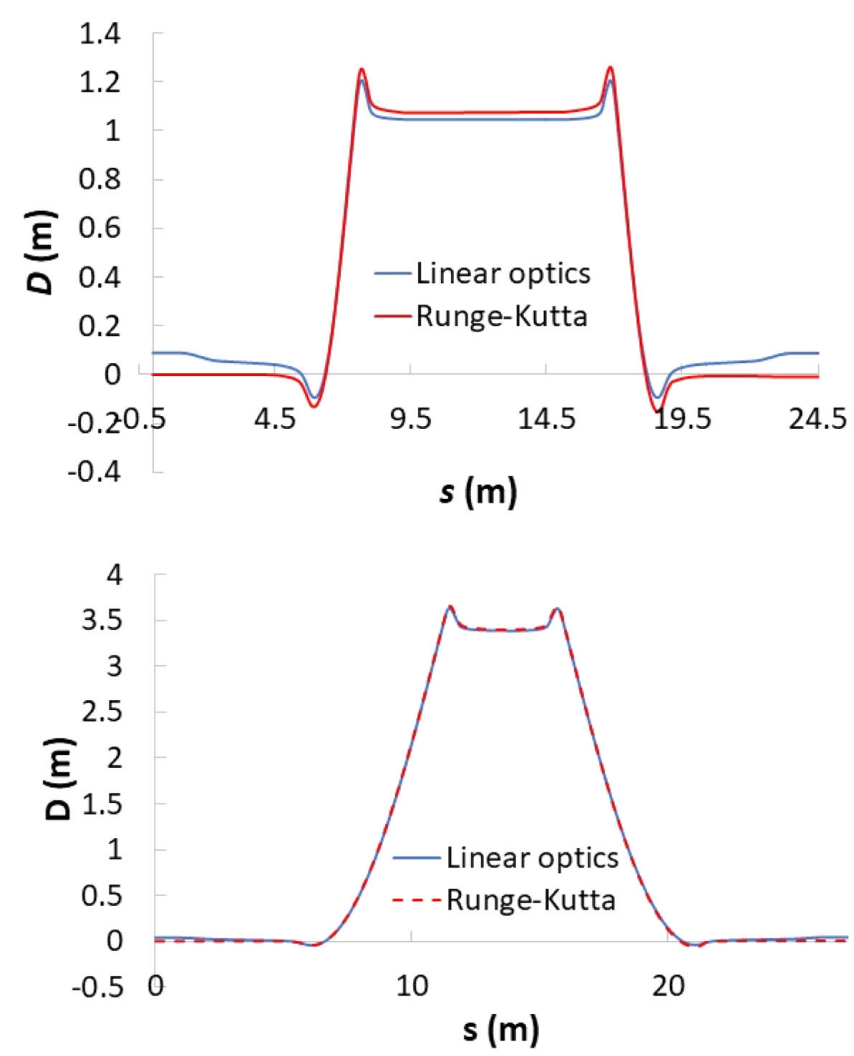

FIG. 14. Dispersion function of the RAFFIA at the injection (upper) and extraction (lower) energy.
$\Delta p / p$ at the injection energy is $0.41 \%$, the maximum $x_{e q}$ is $5.3 \mathrm{~mm}$, which is still sufficiently small. Even though the maximum dispersion function at the extraction energy is almost 3 times bigger than at the injection energy, $\Delta p / p$ at the extraction energy may decrease due to the acceleration. The calculation result also shows that the location of the acceleration device in the straight section has a small dispersion function. Therefore, the emittance blowup caused by synchro-beta coupling can be avoided [16].

\section{Closed-orbit distortion}

The magnetic fields of the actual bending magnet may be different from the magnetic field that is used in the design. This difference is the magnetic field error $\left(\Delta B_{y}\right)$, which generates the COD. In a usual synchrotron, the COD arose from any unpredicted magnetic field imperfections in the accelerator ring and can be minimized by the steering magnet (ST) system in the well-established manner.

In the RAFFIA, an ideal magnetic field that is uniform along the longitudinal direction of the magnet is used in the orbit design. As a matter of fact, a nonuniform magnetic field originated from the finite size of the bending magnet in the $Z$ direction is induced, as shown in the designed magnetic field in Fig. 3. Even though the bending magnet design has been optimized, the nonuniformity still remains more or less. This nonuniform magnetic field is considered as the specific magnetic field error $\left(\Delta B_{y}\right)$ which induces an intrinsic COD. As shown in Fig. 15, a particle experiences a different $\Delta B_{y}$ for each turn, because the particle orbit in the bending magnet varies with acceleration. Therefore, the COD can be considered as the predictable time-varying COD.

In the linear optics approach, the COD can be calculated by the following equation:

$$
\begin{aligned}
X_{\mathrm{COD}}(s)= & \frac{\sqrt{\beta(s)}}{2 \sin (\mu / 2)} * \int_{s}^{s+C}\left\{-\frac{\Delta B_{y}\left(s^{\prime}\right)}{B \rho}\right\} \sqrt{\beta\left(s^{\prime}\right)} \cos \\
& \times\left[\mu / 2-\left|\Psi(s)-\Psi\left(s^{\prime}\right)\right|\right] d s^{\prime} .
\end{aligned}
$$

High-energy orbit

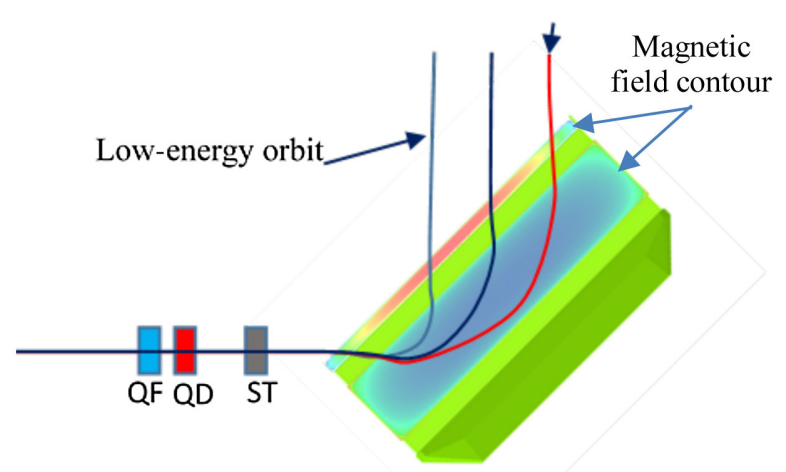

FIG. 15. Magnetic field contour and particle orbits in the bending magnet of the RAFFIA. 


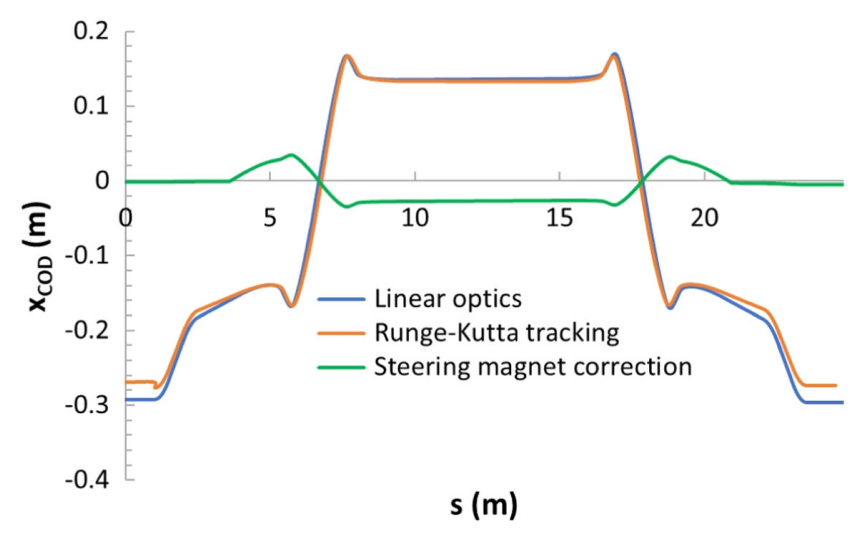

FIG. 16. Bare COD at the injection energy and its corrected one.

On the other hand, the COD is obtained by subtracting that in the case of the ideal magnetic field from a closed orbit in the case of the nonuniform magnetic field. Both closed orbits are obtained by running the particle tracking code with zero emittance. The closed orbit is obtained by scanning the initial position in the phase space. The resultant CODs of the RAFFIA at the injection energy are shown in Fig. 16. The results from the linear optics approach and simulation are in good agreement with each other.

To compensate for the COD, an additional magnetic field is applied by using four steering magnets. For the injection energy, the optimum correction is performed by setting the steering magnet field to $0.0277 \mathrm{~T} \mathrm{~m}$.

\section{Beam dynamics issue: Resonance crossing}

In order to confirm the stable acceleration of cluster ion beams in the transverse direction, the macroparticle tracking of a Gaussian beam over the entire acceleration cycle is carried out, assuming the discrete and constant step acceleration voltage. Figures 17(a) and 17(b) show the temporal evolution of the beam sizes from injection to extraction, where the horizontal beam size changes with a typical adiabatic dumping associated with acceleration and the vertical beam size goes through blowup in the several regions of turns. However, it is noted that any beam loss is not observed in the macroparticle tracking.

To manifest what causes this beam size growth, the temporal evolution of betatron tunes and their footprints is evaluated from the tracking results and shown in Fig. 18. The vertical beam size growth observed around 26, 230, and 970 turns seems to be driven by the fourth-order nonlinear resonance, which is usually originated from the octupole magnetic component. This nonlinear component is likely in the bending magnet with gradient.

One can conclude that the beam size blowup is not fatal and still acceptable without introducing any countermeasure, although it is desired to further reduce the octupole component in the bending magnet design.

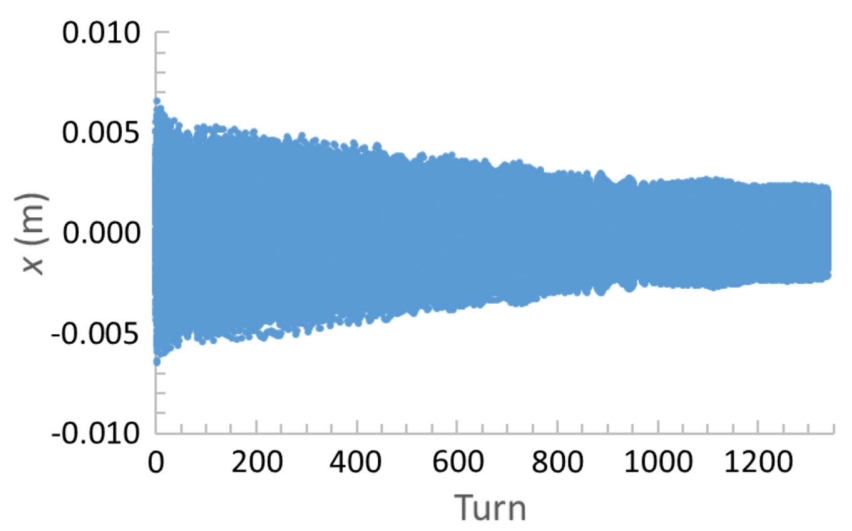

(a)

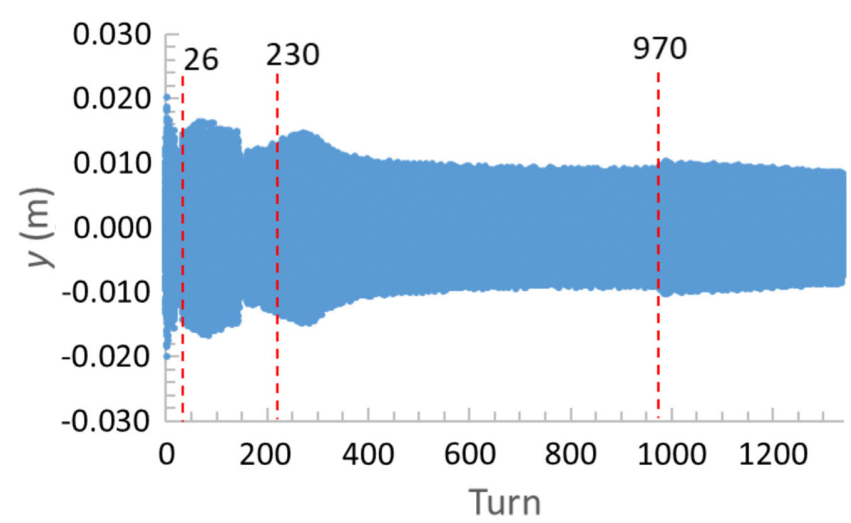

(b)

FIG. 17. The beam size during acceleration in (a) horizontal monitored at the $\mathrm{QF}_{1}$ and (b) vertical monitored at $\mathrm{QD}_{1}$.

\section{LONGITUDINAL MOTION}

\section{A. Injected cluster bunch}

The motion of the particle in the longitudinal direction is mainly determined by the acceleration device. The particles continue to be accelerated and confined by the acceleration voltage $\left(V_{a c}\right)$ and barrier voltage $\left(V_{b b}\right)$. The injected particles are usually distributed in the longitudinal direction with a slightly different energy $(\Delta E)$ and phase $(\phi)$ to the synchronous particle. In this calculation, the energy of the injected particle is assumed to follow a normal distribution with some variance, whereas the initial phase of the particle follows a uniform distribution with a width of $50^{\circ}$.

\section{B. Trapping and acceleration}

The longitudinal motion of the particles that are injected to the RAFFIA are defined by the acceleration equation for the induction accelerator:

$$
\begin{aligned}
(\Delta E)_{n+1} & =(\Delta E)_{n}+q\left[V_{b b}\left(\phi_{n}\right)-V_{a c}\right], \\
\phi_{n+1} & =\phi_{n}+\frac{2 \pi \eta_{n+1}}{\left(\beta_{n+1}^{s}\right)^{2} E_{n+1}^{s}}(\Delta E)_{n+1},
\end{aligned}
$$




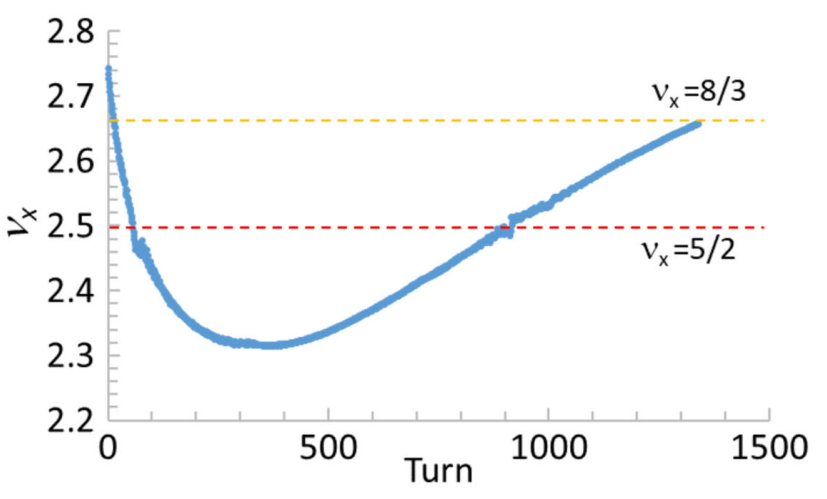

(a)

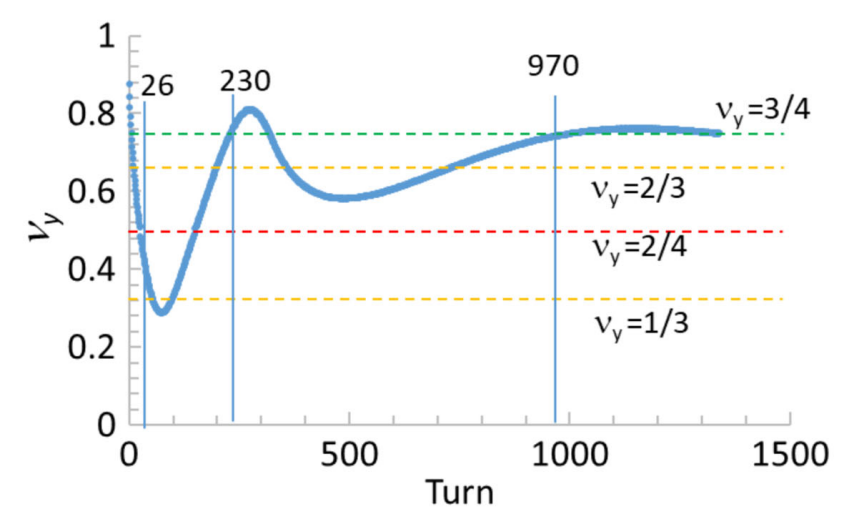

(b)

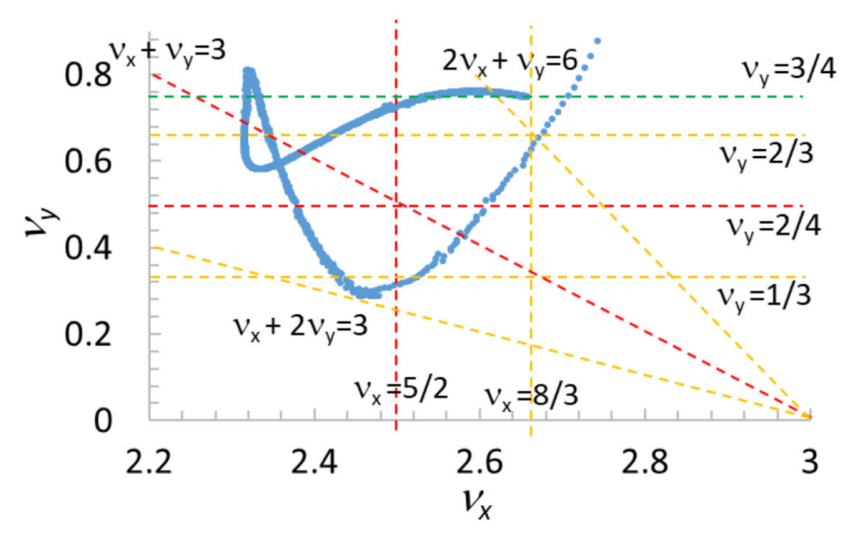

(c)

FIG. 18. (a) Horizontal tune vs turn number, (b) vertical tune vs turn number, and (c) tune footprint.

where $E$ is the particle energy, $n$ is the turn number, $q$ is the particle charge, $\beta$ is the relativistic beta, $\eta=\alpha-\frac{1}{\left(\gamma^{s}\right)^{2}}$ is the slippage factor, $\gamma$ is the Lorentz factor, $\alpha=\frac{1}{C} \oint \frac{D(s)}{\rho} d s$ is the momentum compaction factor, and $C$ is the circumference of the closed orbit. Superscript $s$ denotes the synchronous particle. The calculation result shows that the momentum compaction factor of the RAFFIA increases with particle energy (Fig. 19). This feature of the RAFFIA is different from a synchrotron, which has a constant momentum compaction factor.

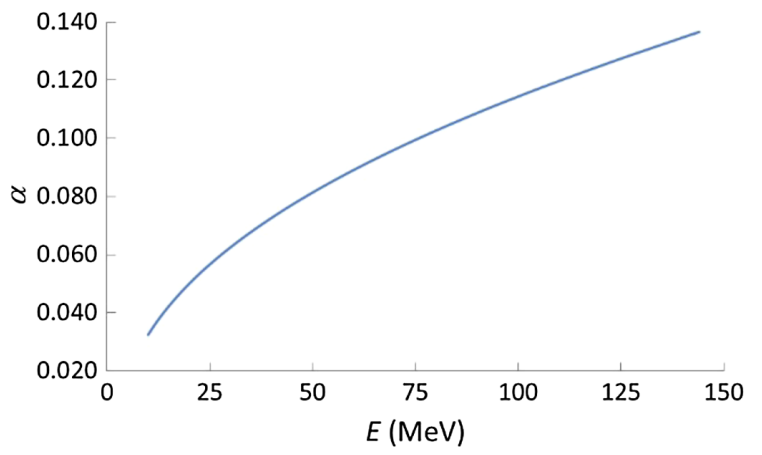

FIG. 19. Momentum compaction factor of the RAFFIA.

In this calculation, we set $V_{b b}$ to $1 \mathrm{kV}$, the phase of the beam center, $\phi_{\text {pulse }}$, to $12^{\circ}$, and the phase distance between two barrier voltages, $\Delta \phi$, to $24^{\circ}$. An induction cell generates a $10 \mathrm{kV}$ acceleration voltage between the two barrier voltage pulses. $\Delta p / p$ and $\phi$ of the injected particles are generated by following a normal distribution and a uniform distribution, respectively. The longitudinal motion is calculated from the injection energy to the extraction energy, and the phase space distributions at the beginning and extraction energy are shown in Fig. 20. The calculation result shows that the momentum deviation decreases at the extraction energy as expected and two barrier voltages confine the beam to a size of $\Delta \phi$. The final longitudinal beam size can be varied by controlling $\Delta \phi$.

\section{Beam loss}

During acceleration, some particles with high $\Delta p / p$ may not be confined. The unconfined particles are lost due to a finite momentum aperture of the RAFFIA ring. The maximum $\Delta p / p$ of the particles that are confined depends on the barrier voltage according to

$$
\left(\frac{\Delta p}{p}\right)_{\max }=\frac{1}{\beta^{s}} \sqrt{\frac{1}{|\eta|}\left(\frac{q V_{b b}}{E^{s}}\right) \frac{2 \tau_{\text {pulse }}}{T_{0}}}
$$

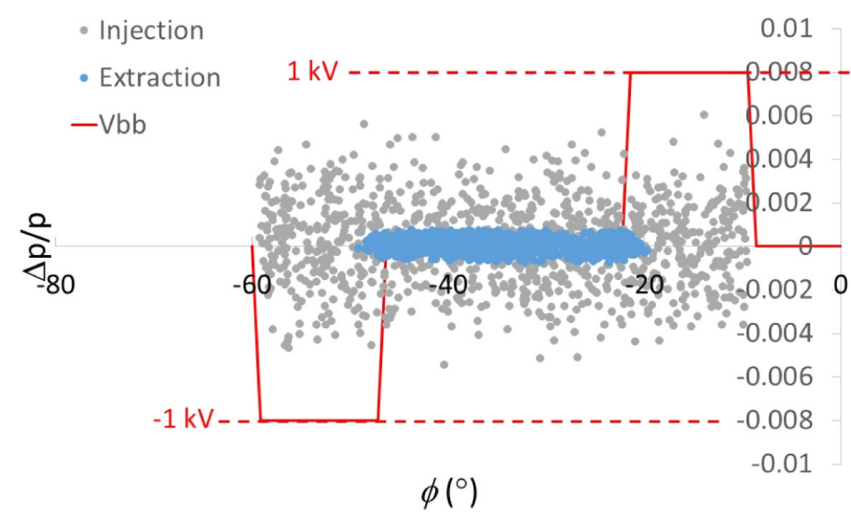

FIG. 20. Longitudinal beam size in the phase space. 


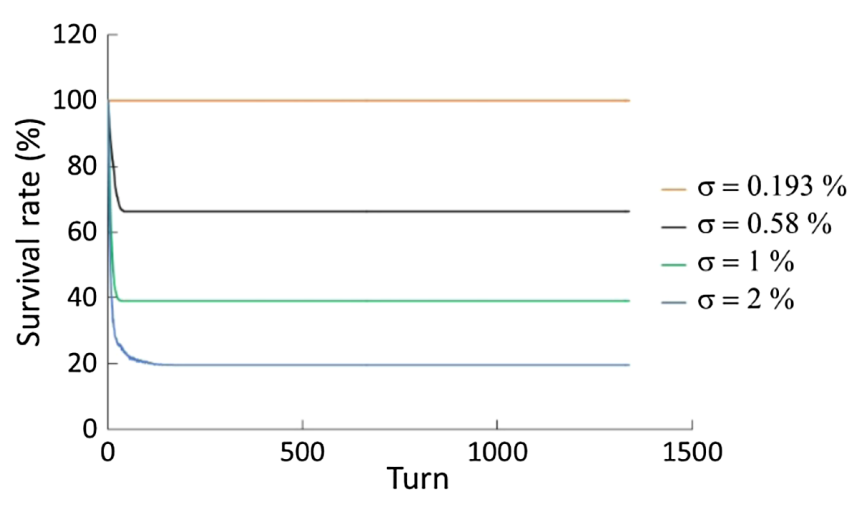

FIG. 21. Survival rate of the ions as a function of $\sigma$ at $\mathrm{V}_{b b}=1 \mathrm{kV}$.

where $\tau_{\text {pulse }}$ is the barrier pulse width and $T_{0}$ is the time period for a single turn. For a barrier voltage of $1 \mathrm{kV}$, $\left(\frac{\Delta p}{p}\right)_{\max }$ is obtained as $0.58 \%$ at the injection energy. Figure 21 shows the survival rate of the particles when the standard deviation $(\sigma)$ of $\Delta p / p$ is varied. Because $\Delta p / p$ decreases at each turn, particle loss occurs only at the early stage of acceleration. At $V_{b b}=1 \mathrm{kV}$, a $100 \%$ survival rate can be achieved at a maximum of $\sigma=0.193 \%$.

\section{REMAINING ISSUES}

In the paper, space-charge effects in both the transverse and longitudinal directions have not been discussed for the purpose to manifest all crucial features of the RAFFIA beam optics that are determined only by the guiding fields. At the injection energy, however, the relativistic beta is quite small because of the large mass to charge ratio of a giant cluster ion. The space-charge effects should strongly affect particle motions, resulting in the space-charge limited current. Our preliminary study suggests the space-charge limited current of around $200 \mu \mathrm{A}$. In addition, the longitudinal space-charge effects may take some role in beam loss from the barrier bucket beyond the conclusion in the present paper. It is known that longitudinal space charge in induction accelerators can be notably mitigated by controlling the barrier voltage pulse profile including its pulse height. The space-charge effects in the RAFFIA is of general concern in our accelerator society, although the RAFFIA for giant cluster ions is not a high-intensity accelerator, and expected and urgent applications of high-energy giant cluster ions in biology science and mutation tend to prefer rather a lower current. Results of its systematic study will be presented in the forthcoming paper.

\section{SUMMARY}

The linear theory of beam dynamics of the RAFFIA and the full Runge-Kutta approach to justify the linear theory have been developed. A stable orbit was obtained by well-optimized ramping of the quadrupole magnets. We expected that the ramping rate of the quadrupole was $15 \mathrm{~Hz}$. The beam envelope in the vertical direction was much bigger than in the horizontal direction, and the maximum beam envelope was located in the defocusing quadrupole section. By using the maximum beam envelope, assuming $1 \times 10^{-5} \mathrm{mrad}$ beam emittance and the maximum deviation from dispersion function of $5.3 \mathrm{~mm}$, the beam size is around $6.5 \mathrm{~cm}$, and the vacuum vessel size is determined to be $10 \mathrm{~cm}$. It turned out that the intrinsic COD caused by the physical limitation of the magnet size can be corrected by programed ramping of a set of the steering magnets. The existence of intrinsic $\mathrm{H}-\mathrm{V}$ coupling in the RAFFIA was identified by the linear theory and the Runge-Kutta full tracking.

The longitudinal motion in the RAFFIA was studied, assuming induction acceleration. The cluster ion bunch was varied by controlling the phase distance between two barrier bucket pulses. The acceptable momentum deviation of the injected particles depended on the barrier bucket pulse height, as expected. It turned out that its longitudinal motion characteristic is almost the same as that of the induction synchrotron [17]; even so, the transition energy changes with acceleration.

Space-charge effects in the RAFFIA are one of most important issues. An acceptable beam intensity may be of big concern for applications. It is well known that the space-charge effects can be estimated with the help of Laslett incoherent tune shift. The substitution of parameters given in this paper into the formula of the Laslett tune shift indicates the space-charge limited current of around $450 \mu \mathrm{A}$. Meanwhile, our recent extensive study on the beam-core instability excited by the space-charge forces [18] shows the much lower threshold current of $200 \mu \mathrm{A}$. Thus, we have supposed that the actual space-charge limited beam current would be determined by the beamcore instability. Even so, the accepted number of cluster ions seems to be sufficiently enough for strongly expected applications such as mutation of microorganisms or creation of novel materials.

More details discussions will be given in a forthcoming paper [19], and more engineering issues such as the vacuum aperture associated with the beam emittances and extraction kicker will be given in a full text of design book.

\section{ACKNOWLEDGMENTS}

This work has been supported by Grants-in-Aid for Scientific Research (B) (KAKENHI 15H03589). One of the authors (Taufik) gratefully acknowledges the program for Research and Innovation in Science and Technology (RISET-Pro) scholarship, provided by the Ministry of Research, Technology and Higher Education, Republic of Indonesia. 


\section{APPENDIX: RUNGE-KUTTA SOLUTION FOR LORENTZ FORCE EQUATION}

$$
\begin{aligned}
\boldsymbol{r}(t+\Delta t)= & \boldsymbol{r}(t)+\frac{\Delta t}{120}\left[11\left(\boldsymbol{k}_{1}+\boldsymbol{k}_{7}\right)+81\left(\boldsymbol{k}_{3}+\boldsymbol{k}_{4}\right)\right. \\
& \left.-32\left(\boldsymbol{k}_{5}+\boldsymbol{k}_{6}\right)\right], \\
\boldsymbol{p}(t+\Delta t)= & \boldsymbol{p}(t)+\frac{\Delta t}{120}\left[11\left(\boldsymbol{k}_{8}+\boldsymbol{k}_{14}\right)+81\left(\boldsymbol{k}_{10}+\boldsymbol{k}_{11}\right)\right. \\
& \left.-32\left(\boldsymbol{k}_{12}+\boldsymbol{k}_{13}\right)\right],
\end{aligned}
$$

where $\boldsymbol{p}, \boldsymbol{r}$, and $\boldsymbol{k}$ are vectors in the global Cartesian coordinate.

Here,

$$
\begin{aligned}
& \boldsymbol{k}_{1}=\boldsymbol{p}_{1} / m \gamma, \quad \boldsymbol{k}_{8}=q\left(\boldsymbol{E}\left(\boldsymbol{r}_{1}\right)+\frac{\boldsymbol{p}_{1} \times \boldsymbol{B}\left(\boldsymbol{r}_{1}\right)}{m \gamma}\right), \\
& \boldsymbol{k}_{2}=\boldsymbol{p}_{2} / m \gamma, \quad \boldsymbol{k}_{9}=q\left(\boldsymbol{E}\left(\boldsymbol{r}_{2}\right)+\frac{\boldsymbol{p}_{2} \times \boldsymbol{B}\left(\boldsymbol{r}_{2}\right)}{m \gamma}\right), \\
& \boldsymbol{k}_{3}=\boldsymbol{p}_{3} / m \gamma, \quad \boldsymbol{k}_{10}=q\left(\boldsymbol{E}\left(\boldsymbol{r}_{3}\right)+\frac{\boldsymbol{p}_{2} \times \boldsymbol{B}\left(\boldsymbol{r}_{3}\right)}{m \gamma}\right), \\
& \boldsymbol{k}_{4}=\boldsymbol{p}_{4} / m \gamma, \quad \boldsymbol{k}_{11}=q\left(\boldsymbol{E}\left(\boldsymbol{r}_{4}\right)+\frac{\boldsymbol{p}_{4} \times \boldsymbol{B}\left(\boldsymbol{r}_{4}\right)}{m \gamma}\right), \\
& \boldsymbol{k}_{5}=\boldsymbol{p}_{5} / m \gamma, \quad \boldsymbol{k}_{12}=q\left(\boldsymbol{E}\left(\boldsymbol{r}_{5}\right)+\frac{\boldsymbol{p}_{5} \times \boldsymbol{B}\left(\boldsymbol{r}_{5}\right)}{m \gamma}\right), \\
& \boldsymbol{k}_{6}=\boldsymbol{p}_{6} / m \gamma, \quad \boldsymbol{k}_{13}=q\left(\boldsymbol{E}\left(\boldsymbol{r}_{6}\right)+\frac{\boldsymbol{p}_{6} \times \boldsymbol{B}\left(\boldsymbol{r}_{6}\right)}{m \gamma}\right), \\
& \boldsymbol{k}_{7}=\boldsymbol{p}_{7} / m \gamma, \\
& \boldsymbol{r}_{1}=\boldsymbol{r}(t), \\
& \boldsymbol{p}_{1}=\boldsymbol{p}(t), \\
& \boldsymbol{r}_{2}=\left(\boldsymbol{E}\left(\boldsymbol{r}_{7}\right)+\frac{\boldsymbol{p}_{7} \times \boldsymbol{B}\left(\boldsymbol{r}_{7}\right)}{m \gamma}\right), \\
& \boldsymbol{p}_{2}=\left(\boldsymbol{p}(t)+\frac{1}{3} \boldsymbol{k}_{1} \Delta t\right), \\
& \boldsymbol{p}_{4}=\left(\boldsymbol{r}(t)+\frac{1}{12}\left(\boldsymbol{k}_{1}+4 t\right),\right. \\
& \boldsymbol{p}_{3}=\left(\boldsymbol{r}(t)+\frac{2}{3} \boldsymbol{k}_{2} \Delta t\right), \\
& \left.\boldsymbol{\boldsymbol { p } _ { 3 }}=(t)+\frac{1}{12}\left(\boldsymbol{k}_{8}+4 \boldsymbol{k}_{9}-\boldsymbol{k}_{10}\right) \Delta t\right), \\
& \left.\boldsymbol{p}_{3} \Delta t\right), \\
& \left.\boldsymbol{p}_{9}\right),
\end{aligned}
$$

$$
\begin{aligned}
& \boldsymbol{r}_{5}=\left(\boldsymbol{r}(t)+\frac{1}{16}\left(-\boldsymbol{k}_{1}+18 \boldsymbol{k}_{2}-3 \boldsymbol{k}_{3}-6 \boldsymbol{k}_{4}\right) \Delta t\right), \\
& \boldsymbol{p}_{5}=\left(\boldsymbol{p}(t)+\frac{1}{16}\left(-\boldsymbol{k}_{8}+18 \boldsymbol{k}_{9}-3 \boldsymbol{k}_{10}-6 \boldsymbol{k}_{11}\right) \Delta t\right), \\
& \boldsymbol{r}_{6}=\left(\boldsymbol{r}(t)+\frac{1}{8}\left(9 \boldsymbol{k}_{2}-3 \boldsymbol{k}_{3}-6 \boldsymbol{k}_{4}+4 \boldsymbol{k}_{5}\right) \Delta t\right), \\
& \boldsymbol{p}_{6}=\left(\boldsymbol{p}(t)+\frac{1}{8}\left(9 \boldsymbol{k}_{9}-3 \boldsymbol{k}_{10}-6 \boldsymbol{k}_{11}+4 \boldsymbol{k}_{12}\right) \Delta t\right), \\
& \boldsymbol{r}_{7}=\left(\boldsymbol{r}(t)+\frac{1}{44}\left(9 \boldsymbol{k}_{1}-36 \boldsymbol{k}_{2}+63 \boldsymbol{k}_{3}+72 \boldsymbol{k}_{4}-64 \boldsymbol{k}_{6}\right) \Delta t\right), \\
& \boldsymbol{p}_{7}=\left(\boldsymbol{p}(t)+\frac{1}{44}\left(9 \boldsymbol{k}_{8}-36 \boldsymbol{k}_{9}+63 \boldsymbol{k}_{10}+72 \boldsymbol{k}_{11}-64 \boldsymbol{k}_{13}\right) \Delta t\right) .
\end{aligned}
$$

[1] I. Yamada and J. Khoury, Cluster ion beam processing: Review of current and prospective applications, MRS Proc. 1354, 11 (2011).

[2] Tsukuba Innovation Area Project on Driver for Giant Cluster Ions, and Their Applications (2017).

[3] K. Horioka, J. Hasegawa, and C. Deutsch (private communication).

[4] P. Attal, S. Della-Negra, D. Gardes, J. D. Larson, Y. Le Beyec, R. Vienet-Legue, and B. Waast, ORION project: acceleration of ion clusters and highly charged biomolecules from $10 \mathrm{MeV}$ to $1 \mathrm{GeV}$, Nucl. Instrum. Methods Phys. Res., Sect. A 328, 293 (1993).

[5] A. E. El-Said, Tracks of $30-\mathrm{MeV} \mathrm{C}$ clusters in yttrium iron garnet studied by scanning force microscopy, Nucl. Instrum. Methods Phys. Res., Sect. B 267, 953 (2009).

[6] H. Dammak, A. Dunlop, D. Lesueur, A. Brunelle, S. Della-Negra, and Y. Le Beyec, Tracks in Metals by MeV Fullerenes, Phys. Rev. Lett. 74, 1135 (1995).

[7] Y. Saitoh, K. Mizuhashi, and S. Tajima, Acceleration of cluster and molecular ions by TIARA $3 \mathrm{MV}$ tandem accelerator, Nucl. Instrum. Methods Phys. Res., Sect. A 452, 61 (2000).

[8] K. Takayama, Y. Arakida, T. Dixit, T. Iwashita, T. Kono, E. Nakamura, K. Otsuka, Y. Shimosaki, K. Torikai, and M. Wake, Experimental Demonstration of the Induction Synchrotron, Phys. Rev. Lett. 98, 054801 (2007).

[9] K. Takayama, T. Yoshimoto, M. Barata, L. K. Wah, X. Liu, T. Iwashita, S. Harada, T. Adachi, T. Arai, D. Arakawa, H. Asao, E. Kadokura, T. Kawakubo, H. Nakanishi, Y. Okada, K. Okamura, K. Okazaki, A. Takagi, S. Takano, and M. Wake, Induction acceleration of heavy ions in the KEK digital accelerator: Demonstration of a fast-cycling induction synchrotron, Phys. Rev. ST Accel. Beams 17, 010101 (2014).

[10] K. Takayama, T. Adachi, M. Wake, and K. Okamura, Racetrack-shape fixed field induction accelerator for giant cluster ions, Phys. Rev. ST Accel. Beams 18, 050101 (2015). 
[11] Taufik T. Adachi, M. Wake, and K. Takayama, Lattice design of the $144 \mathrm{MeV}$ induction microtron for C-60, in Proceedings of the 14th Annual Meeting of Particle Accelerator Society of Japan, Sapporo, Japan, 2017 (Particle accelerator society of Japan, Tokyo, Japan, 2017), pp. 359-363, https://www.pasj.jp/web_publish/ pasj2017/proceedings/.

[12] Discussion within the forum to discuss about a high energy giant cluster ion facility in Japan, 2017.

[13] Taufik, M. Wake, and K. Takayama, A prototype bending magnet with a reverse field strip for the racetrack-shape fixed fields induction accelerator (to be published).

[14] J. C. Butcher, On Runge-Kutta processes of high order, J. Austral. Math. Soc. 4, 179 (1964).

[15] N. Munemoto, S. Takano, E. Kadokura, Taufik T. Yoshimoto X. Liu T. Adachi M. Ikeda T. Kawakubo K. Okamura, K. Takayama, and M. Wake, Direct injection of fully stripped carbon ions into a fast-cycling induction synchrotron and their capture by the barrier bucket, Phys. Rev. Accel. Beams 20, 080101 (2017).

[16] T. Monma, K. Takayama, T. Yoshimoto, and L. Xigguang, Coherent Synchro-Beta Coupling in the KEK Digital Accelerator, in Proceedings of HIAT2015, Yokohama, Japan, 2015 (JACoW, Geneva, Switzerland, 2015), MOPA16, http://accelconf.web.cern.ch/AccelConf/DOI/ HIAT2015/JACoW-HIAT2015-MOPA16.html.

[17] K. Takayama, Induction Accelerators (Springer, Berlin, 2011), Chap. 11.

[18] Taufik and K. Takayama, Beam-core evolution equation and space-charge limit (to be published).

[19] Taufik T. Adachi K. Takayama, and M. Wake, Crucial transverse beam dynamics in the racetrack-shape fixed field induction accelerator for giant cluster ions (to be published). 